\title{
State Determination and Sufficiency of Observables
}

\author{
Katarzyna Lubnauer • Hanna Podsȩdkowska
}

Received: 7 February 2013 / Accepted: 8 May 2013 / Published online: 19 May 2013

(C) The Author(s) 2013. This article is published with open access at Springerlink.com

\begin{abstract}
Informational completeness and the possibility of state distinction and determination are among the more important issues of quantum statistics. We use spectral and semispectral (POV) measures to analyse these questions. For a given $W^{*}$-algebra and a family of normal states on it we investigate the relation between sufficiency in Petz's sense of a $W^{*}$ subalgebra generated by a spectral measure, and the possibility of determination of these states by means of an observable.
\end{abstract}

Keywords Quantum statistic · Weak sufficiency · Discrimination · von Neumann algebra

Mathematics Subject Classification (2000) Primary 46L53 · Secondary 81S05

\section{Introduction}

In the algebraic approach to quantum mechanics a physical system is described by the operator algebra $\mathcal{M}$, and a subalgebra $\mathcal{N}$ of $\mathcal{M}$ is generated by experimental observation, that is by measurement. The information about the states of the system, which are given by the predual of algebra $\mathcal{M}$, after the measurement is limited to the states restricted to subalgebra $\mathcal{N}$. The purpose of measurement is the determination of properties of the physical system under investigation. That is why it is necessary to analyse the $W^{*}$-algebra generated by semispectral measure $e$, representing the measurement, and its sufficiency for family of states "chosen by $e$ ", that means giving good probability measure describing the measurement outcome. We will define them as states determined by $e$.

Sufficiency of a quantum statistic in Petz's sense was introduced in [9] (under the name of 'sufficiency'), and afterwards analyzed in [7, 8]. State determination and distinction was

K. Lubnauer $(\bowtie) \cdot H$. Podsędkowska

Faculty of Mathematics and Computer Sciences, University of Lodz, Ul. S. Banacha 22, 90-238 Łódź, Poland

e-mail: lubnauer@math.uni.lodz.pl

H. Podsędkowska

e-mail: hpodsedk@math.uni.lodz.pl 
investigated in [2] and [1]. Two questions are dealt with in this paper: when the $W^{*}$-algebra generated by spectral or semispectral measure $e$ is sufficient for a family of states $\mathcal{D}_{e}$ (the set of states determined by $e$ ) and what is the characterization of more general set $\mathcal{D}_{\mathcal{N}}$ of all states determined by $W^{*}$-algebra $\mathcal{N}$. In particular, we are interested in the question when the equivalence class $[\rho]_{\mathcal{N}}$ consists of only one element.

\section{Preliminaries, Notation and the Quantum Setup}

Let $\mathcal{H}$ be a Hilbert space with a scalar product $\langle\cdot, \cdot\rangle$. By $\mathcal{P}(\mathcal{H})$ we shall denote the lattice of all orthogonal projections acting in $\mathcal{H} . \mathbb{B}(\mathcal{H})$ will stand for the algebra of all bounded linear operators on $\mathcal{H}$. For $\varphi \in \mathcal{H}$ we shall denote by $P_{[\varphi]}$ the orthogonal projection onto the subspace spanned by $\varphi$ (in Dirac notation $P_{[\varphi]}=|\varphi\rangle\langle\varphi|$ for $\|\varphi\|=1$ ). Let $\mathbb{B}(\mathcal{H})^{+}$denote the set of all self adjoint positive operators from $\mathbb{B}(\mathcal{H})$.

By a von Neumann algebra $\mathcal{M}$ of operators acting on $\mathcal{H}$ we mean a $*$-algebra $\mathcal{M} \subset \mathbb{B}(\mathcal{H})$ which contains the identity operator 1 and closed in the strong operator topology on $\mathbb{B}(\mathcal{H})$, i.e. the topology given by the family of seminorms

$$
\mathbb{B}(\mathcal{H}) \ni x \mapsto\|x \xi\|, \quad \xi \in \mathcal{H} .
$$

For a von Neumann algebra of operators $\mathcal{M}$ acting on a Hilbert space $\mathcal{H}$ we denote by $\mathcal{M}^{\prime}$ the commutant of $\mathcal{M}$, i.e. the algebra of all bounded operators on $\mathcal{H}$ which commute with all the operators from $\mathcal{M}$. In particular, if $\mathcal{M}$ is Abelian then $\mathcal{M} \subset \mathcal{M}^{\prime}$. For basic facts about von Neumann algebras the reader is referred to [5, 6, 13].

The $\sigma$-field of Borel subsets of the real line $\mathbb{R}$ will be denoted by $\mathcal{B}(\mathbb{R})$.

The probability space employed to describe a quantum system consists of a separable Hilbert space $\mathcal{H}$ and a state $\rho$. Assume now $\mathcal{M}=\mathbb{B}(\mathcal{H})$. There is a 1-1 correspondence between normal states $\rho$ on $\mathbb{B}(\mathcal{H})$ and positive operators of trace one $D_{\rho}$ on $\mathcal{H}$, called density matrices, such that

$$
\rho(a)=\operatorname{tr} a D_{\rho}, \quad a \in \mathbb{B}(\mathcal{H}) .
$$

Observables on $\mathcal{H}$ are represented by semispectral measures (POVM) $e$, where $e: \mathcal{B}(\mathbb{R}) \rightarrow$ $\mathbb{B}(\mathcal{H})^{+}$satisfies the conditions:

(i) $0 \leq e(\Delta) \leq \mathbf{1}$ for any $\Delta \in \mathcal{B}(\mathbb{R})$

(ii) $e(\emptyset)=0, e(\mathbb{R})=\mathbf{1}$

(iii) for any pairwise disjoint sets $\Delta_{n} \in \mathcal{B}(\mathbb{R})$,

$$
e\left(\bigcup_{n=1}^{\infty} \Delta_{n}\right)=\sum_{n=1}^{\infty} e\left(\Delta_{n}\right),
$$

where the series converges in the weak operator topology.

For spectral measures $(\mathrm{PVM})$ we will use $E$, where $E: \mathcal{B}(\mathbb{R}) \rightarrow \mathcal{P}(\mathcal{H})$ ( $\equiv$ projection valued measure).

Let $e$ or $E$ be semispectral or spectral measure respectively. For a state $\rho \in \mathcal{M}_{*}$ we can define the probability measure

$$
\Delta \mapsto \rho(e(\Delta))
$$

for any $\Delta \in \mathcal{B}(\mathbb{R})$.

This is interpreted as the probability distribution of measurement outcomes when the system is in the state $\rho$. 


\section{Sufficient Quantum Subalgebra}

The first idea of sufficiency in the noncommutative setting goes back to H. Umegaki (see [11, 12]) and can be described as follows.

Let $\mathcal{M}$ be von Neumann algebra, and $\mathcal{N}$ be subalgebra of $\mathcal{M}$. Thus $\mathcal{N}$ is said to be sufficient in Umegaki's sense for a family of states $\left\{\rho_{\theta}: \theta \in \Theta\right\}$ if there exists a normal conditional expectation $\mathbb{E}: \mathcal{M} \rightarrow \mathcal{N}$ such that for any $\theta \in \Theta$ we have

$$
\rho_{\theta} \circ \mathbb{E}=\rho_{\theta} .
$$

i.e. the states $\rho_{\theta}$ are $\mathbb{E}$-invariant. The requirement that the map $\mathbb{E}: \mathcal{M} \rightarrow \mathcal{N}$ is a normal conditional expectation was afterwards replaced in the papers $[4,9,10]$ by a weaker condition that we have $\alpha: \mathcal{M} \rightarrow \mathcal{N}$ completely (or two)-positive map; nevertheless, the very idea of sufficiency as the possibility of state-invariantly mapping an algebra into its subalgebra remained intact. This replacement leads us to "sufficiency in Petz's sense".

Definition Let $\mathcal{M}$ be a von Neumann algebra and $\left\{\rho_{\theta}: \theta \in \Theta\right\}$ a family of normal states on $\mathcal{M}$. We say that a von Neumann subalgebra $\mathcal{N}$ of the algebra $\mathcal{M}$ is sufficient in Petz's sense for the family $\left\{\rho_{\theta}: \theta \in \Theta\right\}$ if there exists a normal unital two-positive map $\alpha: \mathcal{M} \rightarrow \mathcal{N}$ such that for all $\theta \in \Theta$,

$$
\rho_{\theta} \circ \alpha=\rho_{\theta} .
$$

Sufficiency of algebra $\mathcal{N}$ for the family of states $\left\{\rho_{\theta}\right\}$ was investigated in [7, 8] in some special situation i.e. for

$$
\begin{aligned}
\mathcal{N} & =\mathcal{W}^{*}(E) \\
& =\left\{\Phi(E)=\int_{-\infty}^{\infty} \Phi(\lambda) E(d \lambda): \Phi \text {-a complex-valued bounded Borel function }\right\}
\end{aligned}
$$

where $E$-spectral measure and $\left\{\varphi_{\theta}: \theta \in \Theta\right\}$-vector states (we use $\varphi$ instead $\rho$ for vector states). The result obtained gives a necessary and sufficient condition for $\mathcal{N}$ to be sufficient in Petz's sense for family $\left\{\varphi_{\theta}: \theta \in \Theta\right\}$ :

Theorem 1 Let $\left\{\varphi_{\theta}: \theta \in \Theta\right\}$ be a family of vector states on $\mathbb{B}(\mathcal{H})$ and $\mathcal{N}=\mathcal{W}^{*}(E)$-the von Neumann algebra generated by a spectral measure $E . \mathcal{N}$ is sufficient in Petz's sense for the family $\left\{\varphi_{\theta}: \theta \in \Theta\right\}$ if and only if the following conditions hold:

(i) vectors $\left\{\varphi_{\theta}\right\}_{\theta \in \Theta}$ are pairwise orthogonal, in particular, we have $\left\{\varphi_{\theta}\right\}=\left\{\varphi_{n}\right\}$

(ii) there exists a family of orthogonal projections $E_{n}$ in $\mathcal{N}$, such that $\left|\varphi_{n}\right\rangle\left\langle\varphi_{n}\right| \leq E_{n}$.

Proof Assume that $\mathcal{N}$ is sufficient in Petz's sense, and let $\alpha$ be normal positive unital map $\mathbb{B}(\mathcal{H})$ into $\mathcal{N}$, such that for each $A \in \mathbb{B}(\mathcal{H})$ and for each $\theta \in \Theta$

$$
\varphi_{\theta}(\alpha(A))=\varphi_{\theta}(A)
$$

in Dirac notation:

$$
\left\langle\varphi_{\theta}|\alpha(A)| \varphi_{\theta}\right\rangle=\left\langle\varphi_{\theta}|A| \varphi_{\theta}\right\rangle .
$$


$\mathcal{N}$ is the Abelian algebra generated by spectral measure $E$, thus for each $A \in \mathbb{B}(\mathcal{H})$ there exists a bounded Borel function $\Phi(\cdot ; A)$ such that

$$
\alpha(A)=\int_{-\infty}^{\infty} \Phi(t ; A) E(d t) .
$$

Moreover,

$$
\sup _{t} \operatorname{ess}|\Phi(t ; A)|=\|\alpha(A)\| \leq\|A\| .
$$

For some orthonormal basis of $\mathcal{H}$ denote by $\mathcal{H}_{0}$ the set of all finite linear combinations of elements from this basis with "rational complex" coefficients, that is numbers $a+b i$, such that $a$ and $b$ are rational. Then $\mathcal{H}_{0}$ is a countable dense subset of $\mathcal{H}$, closed with respect to taking sums and multiplying by rational complex numbers. For any $\xi_{1}, \xi_{2}, \xi, \eta_{1}, \eta_{2}, \eta \in \mathcal{H}$ we have

$$
\left(\left|\xi_{1}\right\rangle+\left|\xi_{2}\right\rangle\right)\left\langle\eta|=| \xi_{1}\right\rangle\left\langle\eta|+| \xi_{2}\right\rangle\langle\eta|, \quad| \xi\rangle\left(\left\langle\eta_{1}\left|+\left\langle\eta_{2}\right|\right)=\mid \xi\right\rangle\left\langle\eta_{1}|+| \xi\right\rangle\left\langle\eta_{2}\right|,\right.
$$

so for any $\xi_{1}, \xi_{2}, \eta \in \mathcal{H}_{0}$ we get

$$
\begin{aligned}
\int_{-\infty}^{\infty} \Phi\left(t ;\left(\left|\xi_{1}\right\rangle+\left|\xi_{2}\right\rangle\right)\langle\eta|\right) E(d t) & =\alpha\left(\left(\left|\xi_{1}\right\rangle+\left|\xi_{2}\right\rangle\right)\langle\eta|\right) \\
& =\alpha\left(\left|\xi_{1}\right\rangle\langle\eta|\right)+\alpha\left(\left|\xi_{2}\right\rangle\langle\eta|\right) \\
& =\int_{-\infty}^{\infty}\left[\Phi\left(t ;\left|\xi_{1}\right\rangle\langle\eta|\right)+\Phi\left(t ;\left|\xi_{2}\right\rangle\langle\eta|\right)\right] E(d t)
\end{aligned}
$$

thus

$$
\Phi\left(t ;\left(\left|\xi_{1}\right\rangle+\left|\xi_{2}\right\rangle\right)\langle\eta|\right)=\Phi\left(t ;\left|\xi_{1}\right\rangle\langle\eta|\right)+\Phi\left(t ;\left|\xi_{2}\right\rangle\langle\eta|\right) \quad E-\text { a.e. }
$$

This means that there exists set $\Delta_{\xi_{1}, \xi_{2}, \eta}$ of full $E$-measure, such that for all $t \in \Delta_{\xi_{1}, \xi_{2}, \eta}$ equality is (1) fulfilled. Similarly, with summing on the second place and multiplication by a rational complex number, therefore taking the intersection of all sets of full $E$-measure, we get the set $\Delta_{1}$ of full $E$-measure, such that for all $t \in \Delta_{1}$, for all $\xi_{1}, \xi_{2}, \eta_{1}, \eta_{2} \in \mathcal{H}_{0}$, and for all rational complex numbers $c$ we have

$$
\begin{aligned}
& \Phi\left(t ;\left(\left|\xi_{1}\right\rangle+\left|\xi_{2}\right\rangle\right)\left\langle\eta_{1}\right|\right)=\Phi\left(t ;\left|\xi_{1}\right\rangle\left\langle\eta_{1}\right|\right)+\Phi\left(t ;\left|\xi_{2}\right\rangle\left\langle\eta_{1}\right|\right), \\
& \Phi\left(t ;\left|\xi_{1}\right\rangle\left(\left\langle\eta_{1}\right|+\left\langle\eta_{2}\right|\right)\right)=\Phi\left(t ;\left|\xi_{1}\right\rangle\left\langle\eta_{1}\right|\right)+\Phi\left(t ;\left|\xi_{1}\right\rangle\left\langle\eta_{2}\right|\right), \\
& \Phi\left(t ; c\left|\xi_{1}\right\rangle\left\langle\eta_{1}\right|\right)=c \Phi\left(t ;\left|\xi_{1}\right\rangle\left\langle\eta_{1}\right|\right) .
\end{aligned}
$$

Moreover, for each $\xi \in \mathcal{H}_{0},|\xi\rangle\langle\xi| \geq 0$, thus

$$
0 \leq \alpha(|\xi\rangle\langle\xi|)=\int_{-\infty}^{\infty} \Phi(t ;|\xi\rangle\langle\xi|) E(d t)
$$

so

$$
\Phi(t ;|\xi\rangle\langle\xi|) \geq 0 \quad E-\text { a.e. }
$$

This means that there exists set $\Delta_{\xi}$ of full $E$-measure, such that for all $t \in \Delta_{\xi}$

$$
\Phi(t ;|\xi\rangle\langle\xi|) \geq 0
$$


Putting

$$
\Delta_{2}=\bigcap_{\xi \in \mathcal{H}_{0}} \Delta_{\xi},
$$

we get the set $\Delta_{2}$ of full $E$-measure, such that for all $t \in \Delta_{2}$ and all $\xi \in \mathcal{H}_{0}$

$$
\Phi(t ;|\xi\rangle\langle\xi|) \geq 0 .
$$

For any fixed $\xi, \eta \in \mathcal{H}_{0}$ there is a set $\Delta_{\xi, \eta}$ of full $E$-measure, such that for all $t \in \Delta_{\xi, \eta}$ we have

$$
|\Phi(t ;|\xi\rangle\langle\eta|)| \leq \underset{u}{\sup \operatorname{ess}}|\Phi(u ;|\xi\rangle\langle\eta|)| \leq \||\xi\rangle\langle\eta|\|=\| \xi\|\| \eta \| .
$$

Put

$$
\Delta_{3}=\bigcap_{\xi, \eta \in \mathcal{H}_{0}} \Delta_{\xi, \eta} .
$$

Then $\Delta_{3}$ is of full $E$-measure, and for all $t \in \Delta_{3}$, and for all $\xi, \eta \in \mathcal{H}_{0}$ we have

$$
|\Phi(t ;|\xi\rangle\langle\eta|)| \leq\|\xi\|\|\eta\| \text {. }
$$

Put

$$
\Delta=\Delta_{1} \cap \Delta_{2} \cap \Delta_{3} .
$$

Then $\Delta$ is of full $E$-measure, and for all $t \in \Delta$, and all $\xi_{1}, \xi_{2}, \xi, \eta_{1}, \eta_{2}, \eta \in \mathcal{H}_{0}$, and for all rational complex numbers $c$ relations (2), (3) and (4) hold.

For $t \in \Delta$ we can define function $h(t ; \cdot, \cdot)$ on $\mathcal{H}_{0} \times \mathcal{H}_{0}$ by the formula

$$
h(t ; \xi, \eta)=\Phi(t ;|\xi\rangle\langle\eta|) \text {. }
$$

For all $\xi, \eta \in \mathcal{H}_{0}, h(\cdot ; \xi, \eta)$ is a Borel function, $h(t ; \cdot, \cdot)$ is a sesquilinear form on $\mathcal{H}_{0} \times \mathcal{H}_{0}$ with respect to multiplication by rational complex numbers; moreover

$$
|h(t ; \xi, \eta)| \leq\|\xi\|\|\eta\| \quad \text { and } \quad h(t ; \xi, \xi) \geq 0 .
$$

Let $\mathcal{H}_{0} \ni \xi_{n} \rightarrow \xi, \mathcal{H}_{0} \ni \eta_{n} \rightarrow \eta$. Then for all $t \in \Delta$ we have

$$
\begin{aligned}
& \left|h\left(t ; \xi_{n}, \eta_{n}\right)-h\left(t ; \xi_{m}, \eta_{m}\right)\right| \leq\left|h\left(t ; \xi_{n}, \eta_{n}\right)-h\left(t ; \xi_{n}, \eta_{m}\right)\right| \\
& \quad+\left|h\left(t ; \xi_{n}, \eta_{m}\right)-h\left(t ; \xi_{m}, \eta_{m}\right)\right|=\left|h\left(t ; \xi_{n}, \eta_{n}-\eta_{m}\right)\right|+\left|h\left(t ; \xi_{n}-\xi_{m}, \eta_{m}\right)\right| \\
& \quad \leq\left\|\xi_{n}\right\|\left\|\eta_{n}-\eta_{m}\right\|+\left\|\xi_{n}-\xi_{m}\right\|\left\|\eta_{m}\right\| \underset{n, m \rightarrow \infty}{\longrightarrow} 0,
\end{aligned}
$$

thus for $t \in \Delta$, we can define function $\widetilde{h}(t ; \cdot, \cdot)$ on $\mathcal{H} \times \mathcal{H}$ by the formula

$$
\widetilde{h}(t ; \xi, \eta)=\lim _{n \rightarrow \infty} h\left(t ; \xi_{n}, \eta_{n}\right)
$$

where $\xi_{n}, \eta_{n}$ are as above. Reasoning as in relation (5) above shows, that $\tilde{h}(t ; \xi, \eta)$ doesn't depend on the choice of approximating sequences $\left\{\xi_{n}\right\},\left\{\eta_{n}\right\}$.

Again we find immediately, that for all $\xi, \eta \in \mathcal{H}, \widetilde{h}(\cdot ; \xi, \eta)$ is a Borel function.

It can be easily seen that $\widetilde{h}(t ; \cdot, \cdot)$ is a sesquilinear form on $\mathcal{H} \times \mathcal{H}$ and

$$
|\widetilde{h}(t ; \xi, \eta)|=\lim _{n \rightarrow \infty}\left|h\left(t ; \xi_{n}, \eta_{n}\right)\right| \leq \lim _{n \rightarrow \infty}\left\|\xi_{n}\right\|\left\|\eta_{n}\right\|=\|\xi\|\|\eta\| .
$$


There is, therefore, the operator $A(t)$ of norm $\leq 1$, such that

$$
\langle\eta|A(t)| \xi\rangle=\widetilde{h}(t ; \xi, \eta), \quad \xi, \eta \in \mathcal{H}
$$

moreover, the operator function $t \mapsto A(t)$ is weakly Borel measurable (that is for all $\xi, \eta \in$ $\mathcal{H}, t \mapsto\langle\eta|A(t)| \xi\rangle$ is a Borel function). For $\xi, \eta \in \mathcal{H}_{0}$ we have

$$
\langle\eta|A(t)| \xi\rangle=h(t ; \xi, \eta)=\Phi(t ;|\xi\rangle\langle\eta|) .
$$

In particular, for $\xi \in \mathcal{H}_{0}$ the following inequality holds

$$
\langle\xi|A(t)| \xi\rangle=\Phi(t ;|\xi\rangle\langle\xi|) \geq 0,
$$

showing that

$$
0 \leq A(t) \leq \mathbf{1}
$$

Let now $\varphi$ be an $\alpha$-invariant vector state. Define an operator $A$ (depending on $\varphi$ ) as weak integral

$$
A=\int_{-\infty}^{\infty} A(t)\|E(d t) \varphi\|^{2}
$$

i.e.

$$
\langle\eta|A| \xi\rangle=\int_{-\infty}^{\infty}\langle\eta|A(t)| \xi\rangle\|E(d t) \varphi\|^{2}, \quad \xi, \eta \in \mathcal{H} .
$$

For $\xi, \eta \in \mathcal{H}_{0}$ we have

$$
\begin{aligned}
\langle\eta \mid \varphi\rangle\langle\varphi \mid \xi\rangle & =\langle\varphi \mid \xi\rangle\langle\eta \mid \varphi\rangle \\
& =\langle\varphi|\alpha(|\xi\rangle\langle\eta|)| \varphi\rangle=\int_{-\infty}^{\infty} \Phi(t ;|\xi\rangle\langle\eta|)\|E(d t) \varphi\|^{2} \\
& =\int_{-\infty}^{\infty}\langle\eta|A(t)| \xi\rangle\|E(d t) \varphi\|^{2}=\langle\eta|A| \xi\rangle
\end{aligned}
$$

which shows that

$$
A=|\varphi\rangle\langle\varphi|
$$

Consequently,

$$
1=\langle\varphi|A| \varphi\rangle=\int_{-\infty}^{\infty}\langle\varphi|A(t)| \varphi\rangle\|E(d t) \varphi\|^{2}
$$

and since

$$
\langle\varphi|A(t)| \varphi\rangle \leq 1
$$

we get

$$
\langle\varphi|A(t)| \varphi\rangle=1 \quad\|E(\cdot) \varphi\|^{2}-\text { a.e. }
$$

Taking into account inequalities (7), the above equality yields

$$
A(t) \varphi=\varphi \quad\|E(\cdot) \varphi\|^{2}-\text { a.e. }
$$


For $\xi \perp \varphi$ we have

$$
0=\langle\xi|A| \xi\rangle=\int_{-\infty}^{\infty}\langle\xi|A(t)| \xi\rangle\|E(d t) \varphi\|^{2}
$$

hence

$$
\langle\xi|A(t)| \xi\rangle=0 \quad\|E(\cdot) \varphi\|^{2}-\text { a.e. }
$$

and thus

$$
A(t) \xi=0 \quad\|E(\cdot) \varphi\|^{2}-\text { a.e. }
$$

Consequently, we obtain

$$
A(t)=|\varphi\rangle\langle\varphi| \quad\|E(\cdot) \varphi\|^{2}-\text { a.e. },
$$

thus there is a set $\Delta_{\varphi} \in \mathcal{B}(\mathbb{R})$ such that

$$
\left\|E\left(\Delta_{\varphi}\right) \varphi\right\|^{2}=1
$$

and for all $t \in \Delta_{\varphi}$ we have

$$
A(t)=|\varphi\rangle\langle\varphi| .
$$

From (6) we get for all $\xi, \eta \in \mathcal{H}_{0}$ and all $t \in \Delta \cap \Delta_{\varphi}$

$$
\Phi(t ;|\xi\rangle\langle\eta|)=\langle\eta \mid \varphi\rangle\langle\varphi \mid \xi\rangle=\langle\varphi \mid \xi\rangle\langle\eta \mid \varphi\rangle .
$$

Observe that since $E\left(\Delta_{\varphi}\right)$ is a projection, from equality (8) we get

$$
E\left(\Delta_{\varphi}\right) \varphi=\varphi .
$$

Let now $\varphi$ and $\psi$ be two $\alpha$-invariant vector states. Then we have

$$
\Delta \cap \Delta_{\psi} \cap \Delta_{\varphi}=\emptyset .
$$

Indeed, if $t \in \Delta \cap \Delta_{\psi} \cap \Delta_{\varphi}$, then we would have for $\xi, \eta \in \mathcal{H}_{0}$

$$
\langle\eta \mid \psi\rangle\langle\psi \mid \xi\rangle=\Phi(t ;|\xi\rangle\langle\eta|)=\langle\eta \mid \varphi\rangle\langle\varphi \mid \xi\rangle,
$$

giving the equality $|\psi\rangle\langle\psi|=| \varphi\rangle\langle\varphi|$. In particular, because $\Delta$ is of full $E$-measure, by equality (10) we obtain

$$
\langle\psi \mid \varphi\rangle=\left\langle E\left(\Delta_{\psi}\right) \psi \mid E\left(\Delta_{\varphi}\right) \varphi\right\rangle=\left\langle E\left(\Delta_{\psi} \cap \Delta\right) \psi \mid E\left(\Delta_{\varphi} \cap \Delta\right) \varphi\right\rangle=0,
$$

because from the disjointness of $\Delta_{\varphi} \cap \Delta$ and $\Delta_{\varphi} \cap \Delta$ it follows that

$$
E\left(\Delta_{\varphi} \cap \Delta\right) E\left(\Delta_{\psi} \cap \Delta\right)=0
$$

so two different $\alpha$-invariant pure states are orthogonal.

Let now $\left\{\varphi_{\theta}: \theta \in \Theta\right\}$ be a family of $\alpha$-invariant pure states. Because $\varphi_{\theta}$ are parwise orthogonal, we have $\left\{\varphi_{\theta}\right\}=\left\{\varphi_{\theta_{n}}\right\}$. Let $\Delta_{\varphi_{\theta_{n}}}$ be sets like above. Put

$$
\Delta_{n}=\Delta_{\varphi_{\theta_{n}}} \cap \Delta, \quad E_{n}=E\left(\Delta_{n}\right) .
$$


Sets $\Delta_{n}$ are parwise disjoint, so $E_{n}$ are orthogonal and from (10) we get $\left|\varphi_{\theta_{n}}\right\rangle\left\langle\varphi_{\theta_{n}}\right| \leq E_{n}$.

Assume now, that $\left\{\varphi_{\theta_{n}}\right\}$ is a countable family of orthogonal vector states, which we denote by $\varphi_{n}$, and that there are in $\mathcal{N}$ pairwise orthogonal $E_{n}$ such that $\left|\varphi_{n}\right\rangle\left\langle\varphi_{n}\right| \leq E_{n}$. Put

$$
P=\sum_{n} E_{n}
$$

Let $\psi$ be a fixed vector in $\mathcal{H}$. Define on $\mathbb{B}(\mathcal{H})$ map $\alpha$ by the formula

$$
\alpha(A)=\sum_{n}\left\langle\varphi_{n}|A| \varphi_{n}\right\rangle E_{n}+\langle\psi|A| \psi\rangle P^{\perp}, \quad A \in \mathbb{B}(\mathcal{H})
$$

Of course $\alpha$ is a normal positive unital linear map from $\mathbb{B}(\mathcal{H})$ to $\mathcal{N}$. Since

$$
E_{m} \varphi_{n}= \begin{cases}\varphi_{n}, & \text { if } m=n \\ 0, & \text { if } m \neq n\end{cases}
$$

and

$$
P^{\perp} \varphi_{m}=0 \text { for each } m,
$$

we have

$$
\begin{aligned}
\left\langle\varphi_{n}|\alpha(A)| \varphi_{n}\right\rangle & =\sum_{m}\left\langle\varphi_{m}|A| \varphi_{m}\right\rangle\left\langle\varphi_{n}\left|E_{m}\right| \varphi_{n}\right\rangle+\langle\psi|A| \psi\rangle\left\langle\varphi_{n}\left|P^{\perp}\right| \varphi_{n}\right\rangle \\
& =\left\langle\varphi_{n}|A| \varphi_{n}\right\rangle,
\end{aligned}
$$

for each $n$, which proves that $\varphi_{n}$ are $\alpha$-invariant, so $\mathcal{N}$ is sufficient in Petz's sense (from the commutativity of $\mathcal{N}$ follows completely positivity hence so i two-positivity of $\alpha$ ).

\section{State Distinction and State Determination}

Let us first recall, following [2]

Definition States $\rho$ and $\varphi$ are distinguished by semispectral measure $e$ if

$$
\rho \neq \varphi \quad \Rightarrow \quad \exists \Delta \in \mathcal{B}(\mathbb{R}) \quad \rho(e(\Delta)) \neq \varphi(e(\Delta))
$$

Definition State $\rho$ is determined by semispectral measure $e$ if

$$
\forall \varphi \forall \Delta \in \mathcal{B}(\mathbb{R}) \quad \rho(e(\Delta))=\varphi(e(\Delta)) \quad \Rightarrow \quad \rho=\varphi
$$

For semispectral measure $e$ the equality

$$
\rho(e(\Delta))=\varphi(e(\Delta))
$$

sets up the equivalence relation " $"$ ":

$$
\varphi \sim \rho \quad \Leftrightarrow \quad \forall \Delta \in \mathcal{B}(\mathbb{R}) \quad \rho(e(\Delta))=\varphi(e(\Delta)) .
$$


The implication given in the first of the definitions above

$$
\forall \varphi \forall \Delta \in \mathcal{B}(\mathbb{R}) \quad \rho(e(\Delta))=\varphi(e(\Delta)) \quad \Rightarrow \quad \rho=\varphi
$$

can be rewritten in the following form

$$
[\rho]_{e}=\{\rho\},
$$

where $[\rho]_{e}$ is the equivalence class of relation " ".

The set of all states determined by semispectral measure $e$ will be denoted by $\mathcal{D}_{e}$ and defined by:

$$
\mathcal{D}_{e}=\left\{\rho:[\rho]_{e}=\{\rho\}\right\} .
$$

The content of the set $\mathcal{D}_{e}$ depends on semispectral measure $e$.

For semispectral measures $e$ and $f$, if $\mathcal{D}_{f} \subseteq \mathcal{D}_{e}$ then we say that the state determination power of $e$ is greater than or equal to $f$ and write

$$
\mathcal{D}_{f} \subseteq \mathcal{D}_{e} \Rightarrow f \prec_{d} e
$$

It is known from [1] that for any spectral measure $E$ and any state $\rho$ the equivalence class is a one-element set

$$
[\rho]_{E}=\{\rho\}
$$

if and only if $D_{\rho}$ is a one dimensional projection from $E$, that is $D_{\rho}=|\varphi\rangle\langle\varphi|$ for some unit vector $\varphi \in \mathcal{H}$ (in other worlds, $\rho$ is a vector state).

\section{The Main Problem}

Given subalgebra $\mathcal{N}=W^{*}(e)$ of $\mathcal{M}$ and family of states $\mathcal{D}_{e}$ it is natural to ask if $W^{*}(e)$ is sufficient for $\mathcal{D}_{e}$. The answer is positive for spectral measures and, more general, for randomized semispectral measures, and it is given in the following theorems.

Theorem 2 If $E$ is a spectral measure with values in $\mathcal{P}(\mathcal{H})$, and $\mathcal{D}_{E}$ is the set of states determined by $E$ then $\mathcal{N}=W^{*}(E)$ is sufficient in Petz's sense for the family $\mathcal{D}_{E}$.

Proof On account of $[1,2]$ for spectral measure $E$, set $\mathcal{D}_{E}$ consists only of vector states $\varphi$ such that their density matrices are one-dimensional projections from the range of $E$. So

$$
\mathcal{D}_{E}=\{\varphi:|\varphi\rangle\langle\varphi|=E(\Delta) \text { for some } \Delta \in \mathcal{B}(\mathbb{R})\} .
$$

The Hilbert space $\mathcal{H}$ is separable so there are only countably many such $\varphi$ 's, moreover they are pairwise orthogonal and belong to $\mathcal{N}=W^{*}(E)$. According to Theorem 1 this is a necessary and sufficient condition for $\mathcal{N}$ to be sufficient in Petz's sense for set $\mathcal{D}_{E}$ of states determined by $E$.

Observe that in our situation we can take in formula (11) Theorem $1 E_{n}=\left|\varphi_{n}\right\rangle\left\langle\varphi_{n}\right|$ because $\left|\varphi_{n}\right\rangle\left\langle\varphi_{n}\right| \in W^{*}(E)$.

The same result can be obtained for semispectral randomized measures but we need different methods. The idea of randomized measurements comes from [3]. 
Definition Semispectral measure $e$ with values in $\mathcal{B}(\mathcal{H})^{+}$is called randomized, if for any

$$
\Delta_{1}, \Delta_{2} \in \mathcal{B}(\mathbb{R})
$$

we have

$$
e\left(\Delta_{1}\right) e\left(\Delta_{2}\right)=e\left(\Delta_{2}\right) e\left(\Delta_{1}\right)
$$

The main theorem:

Theorem 3 If $e$ is a randomized semispectral measure and $\mathcal{D}_{e}$ is the set of states determined by e, then the algebra $\mathcal{N}=W^{*}(e)$ is sufficient in Petz's sense for the family $\mathcal{D}_{e}$.

Of course, any spectral measure is a randomized semispectral measure, so the previous theorem follows from this one.

Proof Let $e$ be a semispectral randomized measure. For $\mathcal{N}=W^{*}(e), W^{*}$ we say that two states $\rho$ and $\phi$ are $\mathcal{N}$-equivalent, when for all $A \in \mathcal{N}, \rho(A)=\varphi(A)$. We write $\rho \sim_{\mathcal{N}} \varphi$ and $\sim_{\mathcal{N}}$ is an equivalence relation. In a natural way we define set $\mathcal{D}_{\mathcal{N}}$ of all states determined by algebra $\mathcal{N}$.

$$
\mathcal{D}_{\mathcal{N}}=\left\{\rho:[\rho]_{\mathcal{N}}=\{\rho\}\right\} .
$$

Let's notice that for two algebras $\mathcal{J}$ and $\mathcal{K}$ such that $\mathcal{J} \subset \mathcal{K}$ we have $[\rho]_{\mathcal{J}} \supset[\rho]_{\mathcal{K}}$, indeed for $\varphi \in[\rho]_{\mathcal{K}}$,

$$
\forall_{K \in \mathcal{K}} \quad \varphi(K)=\rho(K)
$$

and from $\mathcal{J} \subset \mathcal{K}$

$$
\forall_{J \in \mathcal{J}} \quad \varphi(J)=\rho(J)
$$

so $\varphi \in[\rho]_{\mathcal{J}}$.

Now it is easy to see that for $\mathcal{J} \subset \mathcal{K}, \mathcal{D}_{\mathcal{J}} \subset \mathcal{D}_{\mathcal{K}}$. By virtue of the assertion above and the fact that $\forall_{\Delta \in \mathcal{B}(\mathbb{R})} e(\Delta) \in \mathcal{N}$ we can claim that $\mathcal{D}_{e} \subset \mathcal{D}_{\mathcal{N}}$.

Each algebra generated by a randomized semispectral measure, like algebra $\mathcal{N}$, is Abelian. On the other hand each Abelian algebra is generated by some spectral measure. Therefore there exists a spectral measure $E$ such that $\mathcal{N}=W^{*}(E)$ and of course $\mathcal{D}_{e} \subset \mathcal{D}_{\mathcal{N}}=\mathcal{D}_{E}$.

$\mathcal{N}=W^{*}(E)$ is sufficient in Petz's sense for the family $\mathcal{D}_{E}$ by Theorem 2, so it is also sufficient for $\mathcal{D}_{e}$ because $\mathcal{D}_{e} \subset \mathcal{D}_{E}$.

\section{Corollary and Open Problems}

There are different kinds of criterions for an optimal measurement. In the first part we have investigated state determination power of a semispectral measure and its correlation with sufficiency but it is also possible to consider distinction power. Following [2] we have:

Definition Let $e, f$ semispectral measure. If for all states $\rho_{1}, \rho_{2}$

$$
\forall \Delta \in \mathcal{B}(\mathbb{R}) \quad \rho_{1}(e(\Delta))=\rho_{2}(e(\Delta)) \quad \Rightarrow \quad \rho_{1}(f(\Delta))=\rho_{2}(f(\Delta))
$$


then we denote

$$
f \prec_{i} e
$$

and say that state distinction power of $e$ is greater or equal to $f$.

We can moreover recall that

$$
f \prec_{i} e \quad \Rightarrow \quad f \prec_{d} e
$$

Corollary 1 If $\mathcal{N}=W^{*}(e)$ is sufficient in Petz's sense for $\mathcal{D}_{e}$ then is sufficient for $\mathcal{D}_{f}$ for each $f$ such that $f \prec_{i}$ e.

The problem of relationship between algebra generated by a spectral measure $e$ and family of states that is determined by $e$ remains unresolved, as well as a structure of $\mathcal{D}_{e}$ for any measurement $\mathrm{e}$ is open issue.

Acknowledgement Work supported by NCN grant no 2011/01/B/ST1/03994.

Open Access This article is distributed under the terms of the Creative Commons Attribution License which permits any use, distribution, and reproduction in any medium, provided the original author(s) and the source are credited.

\section{References}

1. Bush, P., Lahti, P.: The determination of the past and the future of a physical system in quantum mechanics. Found. Phys. 19(6), 633-678 (1989)

2. Heinonen, T.: Optimal measurements in quantum mechanics. Phys. Lett. A 346, 77-86 (2005)

3. Holevo, A.S.: Probabilistic and Statistical Aspects of Quantum Theory. North-Holland Series in Statistics and Probability, vol. 1. North-Holland, Amsterdam (1982)

4. Jenčová, A., Petz, D.: Sufficiency in quantum statistical inference. Commun. Math. Phys. 263, 259-276 (2006)

5. Kadison, R.V., Ringrose, J.R.: Fundamentals of the Theory of Operator Algebras, vol. I. Academic Press, New York (1983)

6. Kadison, R.V., Ringrose, J.R.: Fundamentals of the Theory of Operator Algebras, vol. II. Academic Press, New York (1986)

7. Lubnauer, K., Łuczak, A., Podsȩdkowska, H.: Weak sufficiency of quantum statistics. Rep. Math. Phys. 60, 367-380 (2007)

8. Lubnauer, K., Łuczak, A., Podsędkowska, H.: Weakly sufficient quantum statistics. Math. Slovaca 61, 959-978 (2011)

9. Petz, D.: Sufficient subalgebras and the relative entropy of states of a von Neumann algebra. Commun. Math. Phys. 105, 123-131 (1986)

10. Petz, D.: Sufficiency of channels over von Neumann algebras. Q. J. Math. 39, 907-1008 (1988)

11. Umegaki, H.: Conditional expectation in an operator algebra, III. Kodai Math. Semin. Rep. 11, 51 (1959)

12. Umegaki, H.: Conditional expectation in an operator algebra. IV. Entropy and information). Kodai Math. Semin. Rep. 14, 59 (1962)

13. Takesaki, M.: Theory of Operator Algebras I. Springer, New York (1979) 